\title{
Analyse de la fidélité d'un instrument d'évaluation de la complexité multidimensionnelle (COMID) pour les infirmières à domicile
}

\author{
Analysis of the reliability of a multidimensional complexity scale instrument (COMID) \\ for home care nurses
}

Fanny VALLET, Ph.D, Adjointe scientifique. Institution genevoise de maintien à domicile, Genève, Suisse

Catherine BUSNEL, B.Sc, Responsable de l'unité recherche et développement, Institution genevoise de maintien à domicile, Genève, Suisse

Catherine LUDWIG, Ph.D, Professeure associée Haute école de santé de Genève, Suisse

\begin{abstract}
RÉSUMÉ
Introduction : l'évocation de plus en plus fréquente de la complexité par différents acteurs de santé interroge sur la mesure de ce construit. Le COMID fournit une définition multidimensionnelle et opérationnelle de la complexité et aide les infirmières à domicile au repérage et à l'analyse des situations complexes.

Contexte : une étude d'acceptabilité a montré un intérêt marqué pour le COMID de la part des infirmières, cependant ses propriétés psychométriques n'ont pas encore été examinées.

Objectif : cet article présente deux études testant la fidélité de l'échelle.

Méthode : la première étude porte sur 5401 COMID complétés par des infirmières à domicile dans leur pratique usuelle. La consistance interne a été estimée à l'aide d'un alpha de Cronbach. La seconde étude porte sur 38 COMID, complétés par des infirmières à domicile (juges 1) et des infirmières spécialisées (juges 2). L'accord inter-juges a été examiné par un coefficient de corrélation intra-classe (CCl).

Résultats : le COMID possède une consistance interne acceptable $\mathrm{a}=0.797$ et un accord inter-juges excellent $\mathrm{CCl}=0.839$.

Discussion : les propriétés métrologiques du COMID mériteront d'être complétées par une analyse de sa validité pour le repérage de la complexité dans les soins infirmiers à domicile.

Conclusion : le COMID possède une bonne précision de mesure.
\end{abstract}

Mots clés : complexité, soins à domicile, échelle, validation, fidélité.

ABSTRACT

Introduction: The increasingly frequent mention of complexity by different health care actors challenges the measurement of this construct. The COMID, a tool that helps home nurses to identify complex situations, provides a multidimensional and operational definition of complexity.

Context: While an acceptability study showed that nurses took a keen interest in the COMID, its psychometric properties have not yet been examined.

Objective: This article reports on two studies testing the reliability of the scale.

Method: Study 1 focuses on 5,401 COMIDs completed by home care nurses in their usual practice. The internal consistency was estimated using Cronbach's alpha. Study 2 focuses on 38 COMIDs, completed by home care nurses (rater group 1) and specialized nurses (rater group 2). Inter-rater agreement was examined using an intraclass correlation coefficient (ICC).

Results: The COMID has an acceptable internal consistency $a=0.797$ and an excellent inter-rater agreement $\mathrm{ICC}=0.839$.

Discussion: The metrological properties of the COMID will need to be complemented by an analysis of its validity. Conclusion: The COMID has good measurement accuracy and therefore has good potential for identifying complexity in nursing practice.

Keywords: Complexity, home care, scale, validation, reliability.

Pour citer I'article :

Vallet F, Busnel C, Ludwig C. Analyse de la fidélité d'un instrument d'évaluation de la complexité multidimensionnelle (COMID) pour les infirmières à domicile. Rech Soins Infirm. 2019 Sep;(138):53-64.

Adresse de correspondance :

Fanny Vallet : fanny.vallet@imad-ge.ch 


\section{Remerciements}

Nous remercions les infirmières et les infirmières spécialistes cliniques de l'institution genevoise de maintien à domicile (imad) ayant participé aux études ainsi que Margareth Langard pour son expertise clinique, Claire Pipart et Marc Tanguy pour leur soutien organisationnel et Bruno Moutou pour la logistique des données.

\section{INTRODUCTION}

Les défis des institutions de santé délivrant des prestations de soins à domicile sont nombreux et nécessitent d'être relevés pour permettre à la population de se maintenir et de vieillir à domicile dans les meilleures conditions possibles. Ces défis résultent notamment du vieillissement de la population (1), de l'augmentation des maladies chroniques et de la prévalence de la multimorbidité (2-4), mais aussi du virage ambulatoire (5) qui amène les personnes à réduire leurs séjours hospitaliers au bénéfice de soins à domicile. Dans ce contexte, les professionnels des aides et de soins à domicile se trouvent confrontés à des situations de plus en plus " complexes ", face auxquelles les pratiques doivent s'adapter pour maintenir des soins de qualité, sûrs, efficients, et intégrés s'adaptant aux besoins d'une population vieillissante (6). Or, la prise en charge de situations complexes représente un coût individuel et collectif important : les personnes en situations dites " complexes » consultent davantage en urgence (7), sont plus souvent hospitalisées ou réhospitalisées (8) et restent hospitalisées plus longtemps (9). Les difficultés d'identification ou de gestion des situations " complexes " génèrent une insatisfaction et une incompréhension chez les acteurs de soins (patients, cliniciens, système de soin) (10).

Afin de repérer et prendre en charge de manière optimale une « situation complexe », notamment par une bonne coordination interprofessionnelle, il convient en premier lieu d'en fournir une définition. Bien que la théorie de la complexité ne soit pas encore une théorie pleinement unifiée en raison de sa nature interdisciplinaire (biologie, sociologie, économie, etc.), elle partage certains concepts qui sont la non-linéarité, l'imprévisibilité, les interactions et l'adaptation permanente (11). La discipline infirmière avec Nightingale (12) l'a intégrée très tôt en portant une attention particulière aux relations entre l'humain et son environnement et en favorisant une approche holistique (13). Depuis ces dernières années, différents modèles de soins intégrant la complexité ont été proposés $(14,15)$. Le modèle de prise en soin en a été profondément modifié passant d'un modèle « bio-médical » à un modèle « bio-psycho-social » (16) de plus en plus global et intégré (e.g., Chronic Care Model (17)). En France, la Haute Autorité de Santé définit une situation complexe comme " une situation dans laquelle la présence simultanée d'une multitude de facteurs médicaux, psychosociaux, culturels, environnementaux et/ou économiques sont susceptibles de perturber ou de remettre en cause la prise en charge d'un patient, voire d'aggraver son état de santé » (18). Selon cette définition, la complexité consiste en une accumulation de facteurs liés au patient qui va au-delà des aspects de multimorbidité (19-21) et qui inclut des facteurs médicaux (e.g., douleurs, cognition), socio-économiques (e.g., stresseurs familiaux, littératie), de santé mentale (e.g., dépression, addiction) et comportementaux (e.g., exigences, anxiété liée aux symptômes) (22). À ces aspects liés au patient, s'ajoutent des facteurs d'interférence avec les soins et les prises de décision, d'incertitude du diagnostic, de désorganisation des soins et des aspects relationnels avec le patient (10). Ces différents facteurs n'agissent pas de manière linéaire sur la santé et la prise en charge, mais au contraire, ils les influencent par des processus dynamiques et interactifs $(23,24)$. Par conséquent, il est nécessaire de considérer ces différents facteurs simultanément pour analyser la complexité d'une situation dans sa globalité. Enfin, alors même que la notion de " situation complexe » est largement utilisée au quotidien par les professionnels de la santé et par les décideurs politiques, les éléments significatifs qui rendent une situation complexe sont souvent envisagés différemment selon les contextes (e.g., hospitalier, domiciliaire), les professionnels (e.g., infirmière, médecins généralistes, oncologues, psychiatres, autres médecins spécialistes ou psychologues), les patients et les proches aidants.

Afin de s'accorder sur une évaluation opérationnelle de la complexité, l'utilisation d'un outil d'évaluation standardisée spécifique apparait nécessaire. Les outils développés jusqu'à présent répondaient aux milieux médicaux et hospitaliers, (e.g., I'INTERMED $(25,26)$, ou la Patient-centered assessment method, (PCAM) (27)). Toutefois, de tels instruments font aujourd'hui défaut dans les pratiques domiciliaires et tout particulièrement pour les infirmières qui jouent un rôle essentiel dans le maintien à domicile. En effet, les soins à domicile nécessitent de prendre en compte autant les éléments globaux et systémiques (e.g., proche aidant, adéquation du logement, environnement) que des éléments très spécifiques de santé (e.g., pathologie chronique, aigue, polypathologies) conjointement à une prise en charge intégrée et personnalisée. L'intervention des différents intervenants auprès du patient (e.g., médecin, professionnels de l'aide et des soins et du social, proche aidant) est particulièrement à risque d'entraîner une vision parcellaire et en silo de la situation. Dans un contexte politique favorisant le maintien à 


\section{Analyse de la fidélité d'un instrument d'évaluation de la complexité multidimensionnelle (COMID) pour les infirmières à domicile}

domicile, les infirmières auraient besoin d'être mieux outillées pour identifier les situations complexes en documentant de manière synthétique les éléments multidimensionnels déterminant la complexité. Le défi quotidien est de pouvoir repérer les situations complexes, adapter et préciser les objectifs coordonnés de prise en soin, maintenir la continuité des soins et éviter les hospitalisations inappropriées (28). C'est pour pallier ce manque que l'instrument d'évaluation de la complexité multidimensionnelle (COMID) a été spécifiquement développé pour la pratique infirmière à domicile (29).

Le COMID est un outil construit sous forme de check-list (30 items), couvrant six domaines - ou facteurs - identifiés comme participant à la complexité : 1) la santé médicale, 2) les caractéristiques socio-économiques, 3) la santé mentale, 4) les éléments comportementaux du bénéficiaire de soins, 5) l'instabilité de la situation et 6) les caractéristiques du système de soin. Chacun des six domaines est représenté par 5 items (donnant ainsi un même poids à chaque facteur), pour lesquels l'infirmière juge si le critère est présent « 1 = oui » ou « 0 = non » selon une échelle nominale, permettant de faciliter le repérage des éléments de la complexité. Le score total, qui correspond à la somme des items « présents », peut varier entre 0 et 30 points. Le développement de l'instrument a débuté par l'identification des facteurs de complexité liés au patient (22) et aux soins précédemment identifiés dans la littérature (25) auxquels a été ajouté le facteur d'instabilité. Le COMID a fait l'objet d'une étude d'acceptabilité (29) auprès d'un échantillon de 44 infirmières en soins à domicile. Les résultats ont mis en évidence un intérêt marqué des infirmières pour l'utilisation du COMID dans la pratique de routine, pour sa pertinence et sa simplicité d'utilisation. Cette première étape du processus de validation de l'outil ayant été franchie, une estimation des propriétés métriques de l'instrument devient nécessaire. L'objectif du présent article est de rapporter les résultats de deux études qui ont été menées pour évaluer la fidélité du COMID, c'est-à-dire son degré de précision de mesure. Afin d'évaluer la fidélité de l'instrument, la première étude vise à évaluer sa consistance interne et la seconde étude, la cohérence des jugements de complexité au moyen d'une procédure inter-juges.

\section{PREMIÈRE ÉTUDE : ÉVALUATION DE LA CONSISTANCE INTERNE DU COMID}

L'utilisation du COMID par les infirmières a été implémentée par l'institution genevoise de maintien à domicile (imad) dès fin 2017 pour répondre à des besoins de terrain et dans une perspective de développement et d'adaptation future. Cet outil est utilisé comme support au jugement clinique infirmier sur la complexité de la situation (i.e., sous forme d'une indication retournée à l'infirmière et déterminée à partir du score total) et à son analyse (i.e., évaluation du patient et des coordinations interprofessionnelles). Le COMID peut être complété pour différents motifs : 1) à la suite d'une évaluation standardisée notamment à l'aide du Resident Assessment Instrument Home-
Care (RAl-HC) (30) adapté pour la Suisse - pour les patients de 18 ans et plus bénéficiant de soin à domicile depuis au moins un mois, 2) à la suite d'une réévaluation : trois ou six mois après la précédente évaluation ou lorsque la situation a changé, ou 3) dans le but de préparer une coordination interprofessionnelle. Si le RAI-HC permet une évaluation globale standardisée portant sur 18 domaines de santé, et générant 28 alarmes et quatre échelles de performances (31), il n'intègre pas en première intention le repérage des éléments de la complexité ; d'où le besoin des professionnels d'un outil de repérage pour une utilisation régulière. Pour la première étude, un ensemble de 5401 COMID complétés par des infirmières à domicile dans leur pratique a été considéré.

\section{MÉTHODE}

\section{Procédure de remplissage et récolte des données}

Chaque COMID est rempli par une infirmière ayant une connaissance de la situation de soin : soit l'infirmière est référente de la situation (i.e., l'interlocutrice privilégiée du patient, en charge de la mise à jour et de la qualité du dossier ainsi que de la coordination des soins), soit elle remplace régulièrement la référente ou effectue des soins régulièrement auprès du patient.

Pour les aider dans la cotation du COMID, toutes les infirmières ont à leur disposition un guide donnant des précisions sur la définition des items et leur cotation. Les réponses fournies par l'infirmière sont saisies dans le dossier de soin informatisé via le système MedLink ${ }^{\mathrm{TM}}$ (Medical Link Services SA, Nyon, Suisse). Une fois la complétion du COMID réalisée (suivi d'un retour informatisé), c'est à l'infirmière qu'il revient de qualifier la situation comme simple ou complexe au regard des éléments recueillis et de son analyse clinique.

Les données considérées pour l'étude ont été acquises entre le $1^{\mathrm{er}}$ janvier et le 30 juin 2018 lors d'évaluations cliniques effectuées par des infirmières à domicile auprès de patients de 18 ans et plus recevant des soins depuis au moins un mois. Les données ont été extraites des dossiers informatisés. Dans le cas où plusieurs COMID ont été complétés pour un même patient durant cette période, le dernier COMID de la période a été retenu. Des données complémentaires ont également été extraites telles que l'âge et le sexe des patients faisant l'objet de l'évaluation COMID, mais aussi des données concernant les infirmières qui ont utilisé l'instrument (i.e., âge, sexe, nombre de mois d'ancienneté dans l'institution et si elles étaient les référentes de la situation au moment de la cotation du COMID).

\section{Analyses de données}

Les analyses ont été conduites à l'aide du logiciel SPSS version 23.0 (IBM Corp., Armonk, NY). Sur l'échantillon 
initial de 5401 COMID extraits de la base MedLink, 4994 COMID (soit $92.5 \%$ ) ont été entièrement complétés sans items manquants. Pour $6.1 \%$ des COMID, un seul item était manquant. Toutes les analyses effectuées portent sur l'échantillon total : les données manquantes ont été recodées en les remplaçant par la valeur 0 , considérant une nonréponse comme une absence de problématique ${ }^{1}$.

Dans un premier temps, des analyses descriptives ont été menées pour décrire, d'une part les caractéristiques des infirmières ayant complété les COMID, et d'autre part, les caractéristiques des patients qui ont fait l'objet des évaluations.

Dans un deuxième temps, des analyses descriptives ont été menées pour décrire les réponses obtenues : pourcentage des réponses « oui »/« non » pour chacun des 30 items du COMID, et distribution du score total.

Enfin, dans un troisième temps, la consistance interne du COMID a été évaluée à l'aide d'un alpha de Cronbach (a). Un coefficient supérieur à 0.70 est attendu pour dénoter d'une cohérence interne suffisante, bien qu'un critère plus restrictif de 0.80 soit attendu par certains auteurs (32). Par ailleurs, des corrélations de Bravais-Pearson entre chacun des items et le score total ont été testées. Afin d'attester de la contribution de chacun des items au score total, il est attendu que toutes les corrélations soient significatives.

\section{RÉSULTATS}

\section{Description du collectif d'infirmières et de patients}

Les infirmières ayant complété le COMID sont au nombre de 490. Elles sont âgées de 22 à 64 ans $(M=41.17$, ET = 9.65), ont en moyenne 85.06 mois d'ancienneté dans l'institution (soit plus de 7 années) et $83.7 \%$ sont des femmes. Chaque infirmière a complété de 1 à 42 COMID $(M=11.02)$ pour un total de 5401 COMID. Dans $86.2 \%$ des cas, l'infirmière est référente de la situation.

Les 5401 patients de 18 ans et plus pour lesquels un COMID a été complété sont des femmes dans $62.9 \%$ des cas. Ils sont âgés en moyenne de 78.61 ans $(E T=13.89)$, soit $15.1 \%$

\footnotetext{
1 L'analyse des données manquantes n'a pas mis en évidence de lien entre la présence d'une donnée manquante et des caractéristiques liées au patient ou à l'infirmière : la présence d'une ou plusieurs données manquantes (vs aucune) n'est pas significativement associée au sexe du client $($ chi2 $=0.052, p=0.820)$ ni à son âge $(t(5399)=1.216, p=0.224)$; ni au sexe, à l'âge ou à l'ancienneté au sein de l'institution de maintien à domicile de l'infirmière (respectivement chi2 $=0.665, p=0.415 ; t(5399)=$ $-0.645, p=0.519 ; t(5399)=1.253, p=0.210)$, ni au fait que l'infirmière soit la référente vs ne soit pas la référente du patient (chi2 $=0.042, p=0.838$ ). Par ailleurs, la présence d'une donnée manquante n'est pas liée au score total du COMID $(\mathrm{t}(5399)=1.528, \mathrm{p}=0.127)$.
}

entre 18 et 64 ans, $27.0 \%$ entre 65 et 79 ans et $57.8 \%$ de plus de 80 ans.

\section{Pourcentage des réponses pour chaque item du COMID}

Les pourcentages de réponse « oui » et « non » pour chacun des 30 items du COMID sont rapportés dans le tableau 1. Sur la base de ces données, on constate que les items ayant les pourcentages les plus élevés de réponses « oui » sont la présence de maladies chroniques (64.9\%), la polymédication (62.0\%) et les douleurs chroniques (46.8\%), tous ces items appartenant au domaine de la santé médicale. Les problématiques les moins fréquemment relevées sont relatives à des problèmes d'assurance (2.9\%), d'incohérence thérapeutique (3.1\%) et de changement aigu des capacités cognitives (5.5\%). Enfin, il est important de relever que le pourcentage de valeurs manquantes par item est faible - au plus $0.8 \%$ - ce qui souligne la qualité de complétion du COMID.

\section{Distribution du score total au COMID}

Le score total obtenu au COMID varie de 0 à 25 (voir figure 1). La moyenne est de 6.41 , l'écart-type de 4.35 et la médiane de 6. Plus de deux tiers des scores se trouvent entre les valeurs 2.06 et 10.75 . Le score total présente une légère asymétrie positive (Skew=0.932). Toutefois on ne relève pas d'effet plancher, la valeur 2.06 représentant un écart-type en dessous de la moyenne n'incluant pas le 0 . Ainsi, le score total présente une bonne variabilité.

\section{Consistance interne}

La consistance interne du COMID, évaluée pour l'ensemble des 30 items à l'aide d'un alpha de Cronbach est de $a=0.797$. Ce résultat met en évidence une consistance interne suffisante.

Par ailleurs, chacun des items corrèle significativement avec le score total (de $r=0.175$ à $r=0.543 ; p<0.001$ ). Toutes les corrélations sont positives et relativement homogènes à travers les items (tableau 1). Ainsi, chaque problématique contribue au calcul de la complexité.

\section{DISCUSSION}

Les fréquences des réponses des items montrent que chacune des problématiques évaluées dans le COMID a été observée pour une partie au moins des situations à domicile (i.e., aucune modalité de réponse n'est à $0 \%$ ou $100 \%$ ). Par ailleurs, la distribution des réponses sur le score total montre que ce score est variable entre les situations. Ainsi, de futures analyses pourront s'attacher à identifier si cette variabilité est susceptible de discriminer des situations différentes (analyses de validité). 


\section{Analyse de la fidélité d'un instrument d'évaluation de la complexité multidimensionnelle (COMID) pour les infirmières à domicile}

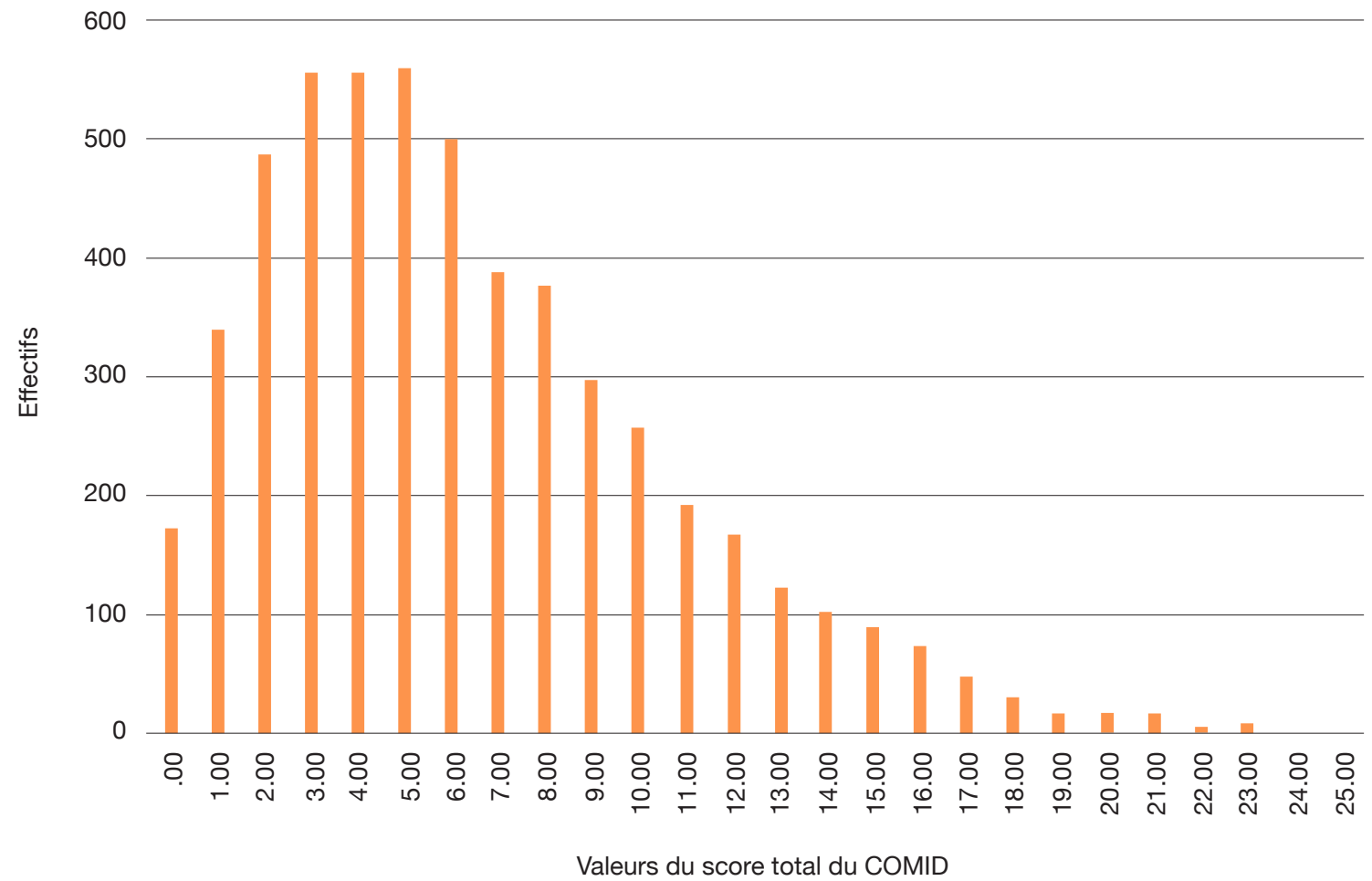

Figure 1 :

Répartition des effectifs pour chaque valeur du score total du COMID

\begin{tabular}{|l|c|c|c|}
\hline \multirow{2}{*}{ Items du CoMID } & \multicolumn{2}{|c|}{ Pourcentages } & \multirow{2}{*}{$\begin{array}{c}\text { Coefficient de corrélations (r) } \\
\text { avec le score total }\end{array}$} \\
\cline { 2 - 3 } & $\%$ « oui » & $\%$ «non » & 0.410 \\
\hline 1a - Maladie chronique & 64.9 & 34.8 & 0.296 \\
\hline 1b - Douleur chronique & 46.8 & 53.0 & 0.175 \\
\hline 1c - Allergie & 14.1 & 85.3 & 0.326 \\
\hline 1d - Polymédication & 62.0 & 37.8 & 0.349 \\
\hline 1e - Troubles cognitifs & 36.6 & 63.1 & 0.410 \\
\hline 2a - Difficultés financières & 12.9 & 86.5 & 0.496 \\
\hline 2b - Absence proche aidant & 33.1 & 66.7 & 0.262 \\
\hline 2c - Faible niveau de littératie & 12.8 & 86.8 & 0.427 \\
\hline 2d - Isolement & 20.2 & 79.5 & 0.336 \\
\hline 2e - Logement inadapté & 8.9 & 90.9 & 0.421 \\
\hline 3a - Dépression & 21.8 & 77.8 & 0.339 \\
\hline 3b - Maladie psychiatrique & 11.8 & 87.8 & 0.293 \\
\hline 3c - Addiction & 9.5 & 90.1 & 0.543 \\
\hline 3d - Anxiété & 21.9 & 77.7 & 0.501 \\
\hline 3e - Variabilité fonctions mentales & 17.1 & 82.4 & 0.473 \\
\hline 4a - Sollicitations récurrentes du réseau & 23.9 & 75.4 & 0.505 \\
\hline 4b - Communication ambivalente & 14.3 & 85.4 & 0.462 \\
\hline 4c - Inquiétude & 26.5 & 73.2 & \\
\hline
\end{tabular}




\begin{tabular}{|l|c|c|c|}
\hline \multirow{2}{*}{ Items du COMID } & \multicolumn{2}{|c|}{ Pourcentages } & \multirow{2}{*}{$\begin{array}{c}\text { Coefficient de corrélations (r) } \\
\text { avec le score total }\end{array}$} \\
\cline { 2 - 3 } & $\%$ « oui » & $\%$ « non » & 0.383 \\
\hline 4d - Agressivité & 7.1 & 92.5 & 0.433 \\
\hline 4e - Résistance aux soins & 15.4 & 84.4 & 0.415 \\
\hline 5a - Dégradation état de santé & 24.4 & 75.4 & 0.321 \\
\hline 5b - Changement degré indépendance & 20.7 & 79.1 & 0.286 \\
\hline 5c - Période transition & 23.1 & 76.5 & 0.289 \\
\hline 5d - Changement aigu capacités cognitives & 5.5 & 94.0 & 0.496 \\
\hline 5e - Non prévisibilité état de santé & 38.1 & 61.6 & 0.458 \\
\hline 6a - Intervenants multiples & 22.9 & 76.7 & 0.353 \\
\hline 6b - Faible partenariat entre intervenants & 9.7 & 89.9 & 0.311 \\
\hline 6c - Incohérence thérapeutique & 3.1 & 96.4 & 0.236 \\
\hline 6d - Problème d'assurance & 2.9 & 96.3 & 0.510 \\
\hline 6e - Ressenti émotionnel lourd & 8.7 & 90.6 & \\
\hline
\end{tabular}

Notes. Dans ce tableau, les réponses « non » ne concernent que les réponses cotées non et pas les réponses manquantes.

a. Toutes les corrélations sont significatives avec un $p<0.001$

\section{Tableau 1: \\ Pourcentage de réponses « oui »/ non » pour chacun des 30 items du COMID et corrélations entre chaque item et le score total}

D'autre part, les analyses mettent en évidence une consistance interne tout à fait acceptable. Toutefois, il est à noter que le COMID est un outil à visée clinique, d'aide au raisonnement, et de soutien à la communication interprofessionnelle. Ainsi, sa précision implique également que deux professionnels différents cotent de manière similaire le COMID. L'évaluation de cet aspect " d'accord inter-juges » de la fidélité du COMID fait l'objet de la seconde étude présentée ci-dessous.

\section{- Seconde étude : évaluation de l'accord inter-juges du COMID}

Pour évaluer le degré d'accord inter-juges du COMID, la seconde étude repose sur un protocole spécifique permettant une double cotation de situations complexes à l'aide de l'outil COMID. Plus spécifiquement, 38 COMID, complétés par une infirmière dans sa pratique, ont été également complétés par un $2^{\text {ème }}$ juge. Cette double cotation a été effectuée par des infirmières spécialistes cliniques (ISC) expérimentées travaillant à l'imad. Les ISC bénéficient d'une solide expérience dans les soins domiciliaires. Elles sont des professionnelles de $2^{\text {ème }}$ ligne, avec une mission de soutien auprès des professionnels des équipes de terrain (infirmier, assistant en soins et santé communautaire, aide familiale, aide à domicile). Elles ont des fonctions de formations, de consultations cliniques spécifiques (e.g., plaies, santé mentale, maladies chroniques) et d'analyses de situations.
Ce protocole impliquant une $2^{\text {ème }}$ cotation du COMID a été mis en place afin de tester l'accord inter-juges pour chacun des 30 items de l'instrument, ainsi que pour le score total. Dans cette étude, seuls les COMID complétés par deux juges selon la procédure définie ont été inclus. Outre l'évaluation de la fidélité, la procédure de l'accord inter-juges permet également, à l'instar de la démarche décrite pour l'INTERMED (33), de repérer d'éventuels items problématiques, à savoir des items pour lesquels l'accord inter-juges est faible.

\section{MÉTHODE}

\section{Procédure de remplissage et récolte
des données}

Les données des COMID complétés par les infirmières à l'imad ont été utilisées pour la cotation de base (juges 1). Au total, 38 situations cliniques ont fait l'objet d'une double cotation. La $2^{\text {ème }}$ cotation (juges 2) a été effectuée par des ISC dans le cadre de formation au RAl-HC assurées auprès de nouvelles infirmières (dans tous types de situations) ou lors de consultations ou d'analyses de situations (dans des situations souvent complexes), entre le $1^{\text {er }}$ décembre 2018 et le 7 février 2019. De manière plus minoritaire, cette $2^{\text {ème }}$ cotation a été effectuée lors d'une demande d'appui pour une analyse de dossier ou d'une préparation de séance de coordination interprofessionnelle. L'intervention des ISC s'est faite auprès de l'infirmière avec ou sans visite au patient. Les doubles cotations du COMID 


\section{Analyse de la fidélité d'un instrument d'évaluation de la complexité multidimensionnelle (COMID) pour les infirmières à domicile}

ont été effectuées de manière indépendante entre les juges (infirmières, juges 1 vs ISC, juges 2) c'est-à-dire sans prendre connaissance des réponses au COMID figurant dans le dossier informatisé du patient. Pour compléter les COMID, les ISC avaient à disposition un ou plusieurs des éléments suivants : le dossier patient informatisé (hors COMID), une visite avec le patient, les informations rapportées par l'infirmière référente ; et plus minoritairement des informations du pôle de gestion de l'équipe ou d'autres collaborateurs ainsi que d'autres sources d'informations documentant la santé du patient. Pour chacune des 38 situations retenues, les COMID des ISC ont été appariés aux COMID saisis dans le dossier informatisé par les infirmières dans leur pratique de routine (complétion des COMID identique à celle décrite dans la première étude). Dans le cas où plusieurs COMID ont été complétés par l'infirmière, le COMID complété dans l'intervalle de temps le plus proche de celui complété par I'ISC a été retenu. L'intervalle de temps entre deux cotations s'étend de 0 à 273 jours ( $M=33.71$ jours, médiane $=13.5$ jours).

\section{Analyses de données}

Les analyses ont été conduites à l'aide du logiciel SPSS version 23.0 (IBM Corp., Armonk, NY). Un total de 38 situations a fait l'objet d'une double cotation.

Dans un premier temps, des analyses descriptives ont été menées pour décrire les caractéristiques des infirmières ayant initialement complété les COMID (juges 1), les caractéristiques des ISC (juges 2) ayant effectué la $2^{\text {ème }}$ cotation, et les caractéristiques des patients qui ont fait l'objet des évaluations.

Dans un second temps, des analyses ont été menées pour évaluer la fidélité inter-juges. Un coefficient de corrélation intra-classe $(\mathrm{CCl})$ a d'abord été calculé pour évaluer le degré d'accord inter-juges sur le score total au COMID. Le modèle utilisé est un modèle mixte à deux facteurs, de type absolu, pour mesure unique (34). Les valeurs sont interprétées en dessous de 0.40 comme faibles; de 0.40 à 0.59 , passables; de 0.60 à 0.74 , bonnes ; et supérieures à 0.75 comme excellentes (35). Des tests Kappa (K) ont ensuite été effectués afin d'évaluer le degré d'accord inter-juges sur les réponses à chacun des 30 items du COMID. Les seuils utilisés pour juger de la qualité des valeurs du coefficient $\mathrm{K}$ sont les suivants (36) : 0.00 à 0.20 , faibles ; de 0.21 à 0.40 , passables ; de 0.41 à 0.60 , modérées ; de 0.61 à 0.80 , considérables, et de 0.81 à 1.00 , excellentes.

\section{RÉSULTATS}

\section{Description du collectif d'infirmières, $\mathrm{d}^{\prime} \mathrm{ISC}$ et de patients}

Trente-cinq infirmières ont complété les COMID qui ont été appariés à ceux complétés par les ISC. Les infirmières sont âgées de 25 à 62 ans $(\mathrm{M}=38.17$, ET = 10.20), 27 sont des femmes, huit des hommes. Elles détiennent en moyenne 58.31 mois d'ancienneté dans l'institution, soit plus de 4 ans en moyenne. Dans 29 situations sur 38, l'infirmière est la référente de la situation.

Dix ISC ont codé indépendamment de 2 à 5 COMID chacune. Les ISC sont âgées de 30 à 58 ans ( $M=45.70, E T=7.50)$, huit sont des femmes et deux des hommes. Elles détiennent en moyenne 121.4 mois d'ancienneté dans l'institution, soit plus de 9 ans en moyenne.

Les patients dont les situations ont été codées sont âgés de 50 à 98 ans $(M=81.06, E T=11.19)$. Vingt-et-un sont des femmes et 17 des hommes.

\section{Accord inter-juges pour le score total du COMID}

Les scores totaux des 38 situations jugées par les infirmières et les ISC sont représentés sous forme de nuages de points dans la figure 2. Le coefficient de corrélation intra-classe calculé sur les valeurs de score total au COMID est de $\mathrm{CCl}=0.839$, avec un intervalle de confiance $(\mathrm{IC})$ à $95 \%$ de $[0.713-0.913]^{2}$. Ce résultat traduit un excellent degré d'accord entre les cotations des ISC et des infirmières.

\section{Accord inter-juges pour les items du COMID}

Les degrés d'accord inter-juges ont été estimés pour chacun des 30 items du COMID, au moyen du calcul de coefficients Kappa (K). Les résultats sont rapportés dans le tableau 2. Ils montrent que quatre items présentent des coefficients d'accord excellents $(\kappa>0.81)$; il s'agit des items de polymédication, difficultés financières, comportement d'agressivité et de résistance aux soins. Inversement, trois items font valoir un accord inter-juges faible ou passable $(\kappa<0.40)$. C'est le cas notamment pour le changement aigu des capacités cognitives et même des accords nonsignificatifs comme le faible partenariat entre intervenants, et l'incohérence thérapeutique. Les autres items ont des accords modérés $(N=13)$ ou considérables $(N=10)$.

\section{DISCUSSION}

Les résultats de l'analyse de 38 doubles cotations du COMID révèlent un accord inter-juges extrêmement élevé sur le score total. L'accord inter-juges évalué sur chacun des items séparément montre des coefficients Kappa qui s'étendent de $\mathrm{K}=0.91$ pour la polymédication à $\mathrm{K}=0.08$ pour le partenariat

\footnotetext{
2 II est à noter que le coefficient de corrélation de Bravais-Pearson entre les scores totaux des COMID cotés par les infirmières et des ISC est de $r=0.854, p<0.001$
} 


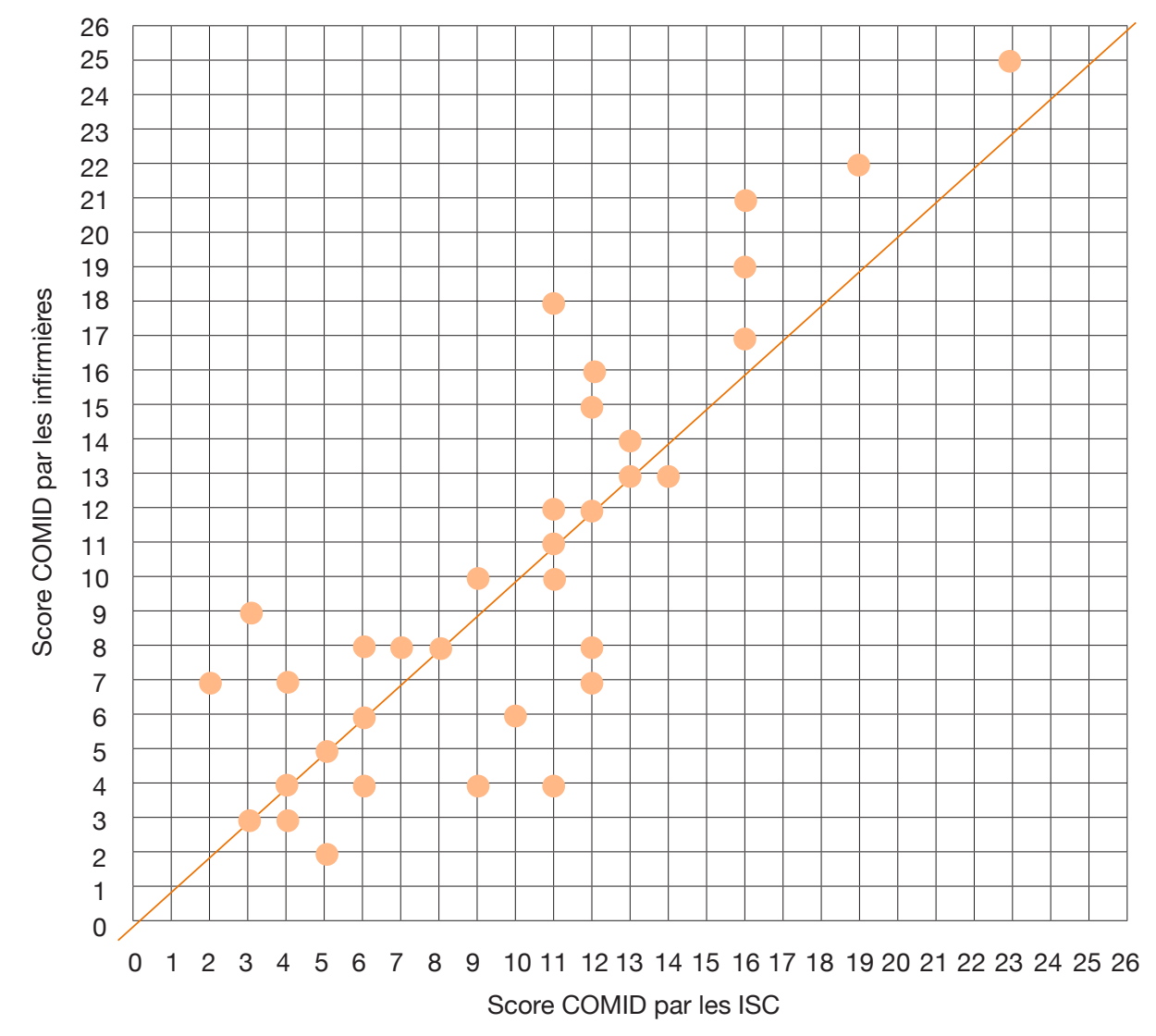

Note. La ligne orange représente la droite théorique d'un accord parfait.

Figure 2 :

Nuage de points représentant les valeurs des scores totaux au COMID codés par les infirmières et les infirmières spécialisées (ISC)

\begin{tabular}{|c|c|c|c|c|}
\hline Items & Kappas $^{a}$ & $p$ & $\begin{array}{l}\text { INF, Réponses } \\
\text { " oui " } \\
\%(\mathrm{~N}) \\
\end{array}$ & $\begin{array}{c}\text { ISC, Réponses } \\
\text { " oui » } \\
\%(\mathrm{~N})\end{array}$ \\
\hline 1a - Maladie chronique & $0.710^{\star \star}$ & $<0.001$ & $73.7(28 / 38)$ & $78.9(30 / 38)$ \\
\hline 1b - Douleur chronique & $0.579^{*}$ & $<0.001$ & $50(19 / 38)$ & $50(19 / 38)$ \\
\hline 1c - Allergie & $0.723^{\star \star}$ & $<0.001$ & $13.2(5 / 38)$ & $7.9(3 / 38)$ \\
\hline 1d - Polymédication & $0.907^{\star * *}$ & $<0.001$ & $81.6(31 / 38)$ & $84.2(32 / 38)$ \\
\hline 1e - Troubles cognitifs & $0.681^{\star \star}$ & $<0.001$ & $55.3(21 / 38)$ & $55.3(21 / 38)$ \\
\hline 2a - Difficultés financières & $0.864^{\star * \star}$ & $<0.001$ & $26.3(10 / 38)$ & $26.3(10 / 38)$ \\
\hline $2 \mathrm{~b}$ - Absence proche aidant & $0.632^{\star \star}$ & $<0.001$ & $50(19 / 38)$ & $47.4(18 / 38)$ \\
\hline 2c - Faible niveau de littératie & $0.541^{*}$ & 0.001 & $21.1(8 / 38)$ & $13.2(5 / 38)$ \\
\hline $2 \mathrm{~d}$ - Isolement & $0.516^{*}$ & 0.001 & $47.4(18 / 38)$ & $28.9(11 / 38)$ \\
\hline 2e - Logement inadapté & $0.791^{\star \star}$ & $<0.001$ & $28.9(11 / 38)$ & $21.1(8 / 38)$ \\
\hline 3a - Dépression & $0.691^{\star *}$ & $<0.001$ & $26.3(10 / 38)$ & $34.2(13 / 38)$ \\
\hline 3b - Maladie psychiatrique & $0.802^{\star \star}$ & $<0.001$ & $15.8(6 / 38)$ & $15.8(6 / 38)$ \\
\hline 3c - Addiction & $0.623^{\star \star}$ & $<0.001$ & $10.5(4 / 38)$ & $13.2(5 / 38)$ \\
\hline 3d - Anxiété & $0.538^{\star}$ & 0.001 & $39.5(15 / 38)$ & $28.9(11 / 38)$ \\
\hline $3 e-$ Variabilité fonctions mentales & $0.543^{\star}$ & 0.001 & $31.6(12 / 38)$ & $23.7(9 / 38)$ \\
\hline
\end{tabular}




\section{Analyse de la fidélité d'un instrument d'évaluation de la complexité multidimensionnelle (COMID) pour les infirmières à domicile}

\begin{tabular}{|c|c|c|c|c|}
\hline Items & Kappas $^{a}$ & $p$ & $\begin{array}{l}\text { INF, Réponses } \\
\text { "oui " } \\
\% \text { (N) }\end{array}$ & $\begin{array}{c}\text { ISC, Réponses } \\
\text { " oui " } \\
\%(\mathrm{~N})\end{array}$ \\
\hline 4a - Sollicitations récurrentes du réseau & $0.463^{*}$ & 0.003 & $47.4(18 / 38)$ & $31.6(12 / 38)$ \\
\hline 4b-Communication ambivalente & $0.662^{* \star}$ & $<0.001$ & $39.5(15 / 38)$ & $34.2(13 / 38)$ \\
\hline 4c - Inquiétude & $0.490^{*}$ & 0.002 & $31.6(12 / 38)$ & $26.3(10 / 38)$ \\
\hline 4d - Agressivité & $0.894^{\star \star \star}$ & $<0.001$ & $15.8(6 / 38)$ & $13.2(5 / 38)$ \\
\hline 4e - Résistance aux soins & $0.841^{* \star *}$ & $<0.001$ & $44.7(17 / 38)$ & $47.4(18 / 38)$ \\
\hline 5a - Dégradation état de santé & $0.474^{*}$ & 0.002 & $26.3(10 / 38)$ & $39.5(15 / 38)$ \\
\hline $5 \mathrm{~b}$ - Changement degré indépendance & $0.549^{*}$ & $<0.001$ & $21.1(8 / 38)$ & $34.2(13 / 38)$ \\
\hline $5 \mathrm{c}$ - Période transition & $0.418^{*}$ & 0.01 & $23.7(9 / 38)$ & $23.7(9 / 38)$ \\
\hline $5 d$ - Changement aigu capacités cognitives & 0.374 & 0.003 & $10.5(4 / 38)$ & $2.6(1 / 38)$ \\
\hline $5 e$ - Non prévisibilité état de santé & $0.575^{\star}$ & $<0.001$ & $52.6(20 / 38)$ & $57.9(22 / 38)$ \\
\hline 6a - Intervenants multiples & $0.411^{*}$ & 0.011 & $44.7(17 / 38)$ & $42.1(16 / 38)$ \\
\hline $6 \mathrm{~b}$ - Faible partenariat entre intervenants & 0.084 & 0.593 & $10.5(4 / 38)$ & $15.8(6 / 38)$ \\
\hline 6c - Incohérence thérapeutique & 0.168 & 0.281 & $13.2(5 / 38)$ & $7.9(3 / 38)$ \\
\hline 6d - Problème d'assurance & $0.479^{*}$ & 0.001 & $7.9(3 / 38)$ & $2.6(1 / 38)$ \\
\hline 6e - Ressenti émotionnel lourd & $0.734^{\star \star}$ & $<0.001$ & $39.5(15 / 38)$ & $47.4(18 / 38)$ \\
\hline
\end{tabular}

Note. a. * : Kappa entre 0.41 et 0.60 , interprété comme modéré ; ${ }^{\star *}$ : Kappa entre 0.61 et 0.80 interprété comme considérable ;

${ }^{* * *}$ : Kappa supérieur à 0.81 interprété comme excellent (36).

Tableau 2 :

Coefficients Kappas et pourcentage de réponses « oui » cotées au COMID par les infirmières (INF) et par les infirmières spécialisées (ISC) pour chaque item

entre intervenants. À noter que cet item, tout comme l'item relatif à l'incohérence thérapeutique $(K=0.17)$ sont les seuls à ne pas atteindre le seuil de significativité de $p<0.05$. Globalement, on peut retenir que la fidélité du COMID, estimée sur la base de méthodes évaluant l'accord inter-juges, est tout à fait acceptable.

Pour ce qui concerne les items «problématiques ", à savoir ceux avec un accord faible ou modéré $(k<0.60)$, on peut relever qu'il s'agit d'items qui évaluent des éléments nécessitant une connaissance approfondie de la situation. Cela pourrait expliquer les cas où le pourcentage de réponses " oui » est plus élevé chez les infirmières que chez les ISC (47.4\% vs $28.9 \%$, par exemple, pour l'isolement, ou $47.4 \%$ vs $31.6 \%$ pour la sollicitation récurrente du réseau). Lors des rencontres et des échanges réguliers avec le patient, elles peuvent observer des éléments de la situation qui ne sont pas forcément visibles dans un premier temps ou documentés en tant que tels, dans le dossier du patient. Cela peut être le cas également par exemple pour la variabilité des fonctions mentales ou des problèmes d'assurance.

Dans d'autres cas, l'expertise de I'ISC peut amener à juger un item plus fréquemment comme " complexe » que l'infirmière, comme c'est le cas par exemple pour l'item concernant le faible partenariat entre intervenants (10.5\% vs $15.8 \%$ ) ou la dégradation de l'état de santé
(26.3\% vs $39.5 \%$ ). Pour d'autres items (e.g., période de transition), l'accord n'est pas élevé sans que cela puisse s'expliquer par le fait que l'un ou l'autre des juges ait plus fréquemment rapporté cette problématique (e.g., $23.7 \%$ dans les deux cas). Par ailleurs, d'autres items, notamment ceux relevant du domaine 6 concernant les intervenants et le système de soins (e.g., l'incohérence thérapeutique), laissent place à une part de subjectivité du professionnel, (e.g., en fonction de son expérience, de sa relation avec le patient), ce qui pourrait être à l'origine d'un plus faible accord entre les deux cotations.

Pour remédier au faible degré d'accord sur certains items, il serait envisageable de favoriser dans la pratique la mise en exergue d'informations saillantes dans le dossier patient. Un autre axe d'amélioration possible serait d'ajouter dans le guide d'utilisation du COMID des explications ou des précisions supplémentaires sur certains items, leur définition et leur cotation. Dans le sens de cette démarche, pour l'outil INTERMED la fidélité des items problématiques a été améliorée, en partie, en ajoutant des précisions dans la formulation des items (e.g., distinguer les pronostics à court terme ou à long terme (33)). On peut aussi relever que pour le COMID, les items qui présentent un haut degré d'accord inter-juges (e.g., les items qui évaluent les problématiques relatives à la résistance aux soins, à l'agressivité et à la prise de médicaments), reposent sur 
des éléments documentés lors de l'évaluation des besoins de santé effectuée avec le RAI-HC. Au final, quelle que soit l'information utilisée par les professionnelles pour compléter le COMID, les résultats de la seconde étude mettent en avant que la majorité des items du COMID sont suffisamment précis pour permettre des réponses similaires entre deux infirmières ayant pourtant des rôles différents et des actions et des fonctions différentes auprès du patient.

Enfin, cette seconde étude présente certaines limites à prendre en compte dans des études ultérieures. La première est l'intervalle de temps entre deux COMID. Celui-ci est très variable, allant d'une double cotation effectuée le même jour à un intervalle de plusieurs mois entre deux cotations d'une même situation. Dans ce dernier cas, il est possible que certaines problématiques aient évolué entre deux occasions de cotation, diminuant ainsi le degré d'accord inter-juges. Par définition, on peut ains attendre qu'un long intervalle entre deux cotations affecte plus particulièrement les cotations aux items évaluant la dimension d'instabilité. La seconde limite tient aux types de situations de cette deuxième étude qui sont plus complexes que dans la première étude (i.e., score total plus élevé, fréquences de certains items plus élevées comme la polymédication, les troubles cognitifs, l'isolement, les sollicitations récurrentes du réseau, la non prévisibilité de l'état de santé ou l'incohérence thérapeutique). Les situations rencontrées par les ISC et ayant fait l'objet de doubles cotations sont donc particulièrement complexes et probablement plus spécifiques que l'ensemble des COMID analysés dans la première étude. Enfin, le nombre de situations ( $N=38$ ) mériterait d'être augmenté pour de meilleures estimations de l'accord inter-juges. Par ailleurs, ces résultats pourraient être affinés afin de voir si l'accord inter-juges varie, par exemple, en fonction du nombre de visites ou de l'expérience professionnelle.

\section{DISCUSSION GÉNÉRALE}

Les deux études présentées dans cet article ont eu pour objectif de documenter la fidélité du COMID, ouvrant ainsi l'évaluation métrologique de l'instrument. Les résultats viennent compléter une première étude qui s'est intéressée à l'acceptabilité du COMID auprès des infirmières à domicile et qui avait donné lieu à des résultats positifs. Si l'on en croit le nombre important de COMID pleinement renseignés depuis son implémentation, l'intérêt porté à l'instrument dans la pratique semble répliqué. En effet, comme le montre la première étude qui a porté sur 5401 COMID complétés par 490 infirmières, rares sont les protocoles qui comportent des données manquantes. Par ailleurs, le nombre important de COMID renseignés d'une part et la variabilité des caractéristiques des patients dont les situations ont été étudiées d'autre part, amènent à penser que ces résultats pourraient être transférables à d'autres échantillons bien que des analyses dans de futures études soient nécessaires pour pouvoir l'affirmer.

On peut également relever qu'un des apports du présent article est d'avoir évalué la fidélité de l'instrument au moyen de deux méthodes, l'une rapportée dans la première étude et visant à estimer la consistance interne de l'instrument, et la seconde, rapportée dans la seconde étude, visant à s'appuyer sur l'accord-inter juges. Les résultats permettent d'inscrire le COMID parmi les outils de mesure de la complexité présentant un bon taux de complétion et une bonne fidélité (pour des résultats similaires avec INTERMED $(33,37)$ ou INTERMED pour des personnes âgées (38). Ils soutiennent ainsi l'utilisation du COMID comme un instrument fidèle pour mesurer la complexité des situations rencontrées à domicile. Les résultats de ces études suggèrent également que la fidélité repose en partie sur les connaissances et l'expérience des infirmières qui leur permettent d'affiner leurs observations. Afin de perfectionner la précision de cotation du COMID, une révision du guide, portant notamment sur la clarté des consignes et des éléments jugés comme " complexes ", ainsi qu'une adaptation de la formation des utilisateurs peuvent être proposés.

Des études complémentaires mériteront d'être menées pour affiner les connaissances métrologiques de l'instrument. II s'agira notamment d'évaluer la validité du COMID, c'est-à-dire sa capacité à effectivement mesurer la complexité multidimensionnelle à domicile. La validité concurrente pourra être estimée en confrontant les réponses obtenues au COMID avec une évaluation de la complexité fondée sur le jugement clinique infirmier. À l'inverse, la validité discriminante pourra être évaluée en confrontant le COMID à des mesures en lien mais dont les construits sont au demeurant distincts de la complexité, tels que la fragilité, les comorbidités ou la dépendance fonctionnelle. Par ailleurs, il serait pertinent de mener des études (39) permettant d'évaluer la validité prédictive de la complexité évaluée à l'aide du COMID. Pour cela, un devis de recherche longitudinal, documentant de manière prospective les évènements indésirables en santé (e.g., suivi des hospitalisations, réhospitalisations, institutionnalisation) devrait être mis en place. Les études pourraient également être déclinées dans une visée de détermination de différents seuils de conversion (courbes $\mathrm{ROC}$ ) de l'instrument mais aussi de précision diagnostique (sensibilité, spécificité) en regard de la complexité. Enfin, la suite du processus de validation du COMID visera à étudier la validité incrémentielle concernant la plus-value de l'utilisation de l'instrument en testant plus précisément 1) l'impact de l'introduction du COMID dans les pratiques professionnelles quotidiennes et 2) la prise en charge des patients selon leur profil de réponse au COMID en terme d'évolution des indicateurs de santé et de prise en charge (e.g., coordinations, délégations de situations simples). 


\section{Analyse de la fidélité d'un instrument d'évaluation de la complexité multidimensionnelle (COMID) pour les infirmières à domicile}

\section{CONCLUSION}

Les deux études présentées dans cet article ont montré que le COMID est un outil d'évaluation de la complexité multidimensionnelle à domicile fidèle. Sa particularité est d'avoir été conçu spécifiquement pour les infirmières intervenant à domicile, qui se trouvent au centre d'un système complexe composé du patient dans son environnement et dans lequel interviennent de multiples professionnels de santé. Le COMID est ainsi un outil particulièrement novateur car, à notre connaissance, il est le premier à être conçu spécifiquement pour les situations prises en soins dans un contexte domiciliaire, et à permettre d'évaluer simultanément différents aspects de la complexité pour les inscrire dans une démarche de prise en soins globale, intégrée, individualisée et adaptée aux besoins des patients.

Au-delà de l'utilisation du COMID dans la démarche de soin (repérage et analyse des situations complexes permettant d'affiner les coordinations et les plans d'intervention), les données issues du COMID, prises à l'échelle d'une institution, constituent également une source précieuse d'informations additionnelles pour améliorer la connaissance de la clientèle (31). Ainsi, en se fondant sur le COMID, l'analyse de la complexité peut se faire à un niveau " micro " (en évaluant les caractéristiques et l'environnement du patient), au niveau “ méso » (en évaluant les ressources nécessaires pour répondre aux besoins de patients) et au niveau « macro » (en évaluant la complexité des situations d'une population donnée et dans les coordinations mises en place dans le système de santé). Dans la pratique clinique quotidienne, une analyse de la complexité à ces différents niveaux est indispensable pour communiquer, coordonner et proposer une prise en charge de qualité.

\section{FINANCEMENTS}

L'étude a été soutenue par des fonds institutionnels de l'institution genevoise de maintien à domicile (imad). Le financeur n'a joué aucun rôle dans l'élaboration du plan recherche, dans le traitement des données et dans la préparation du manuscrit.

\section{DÉCLARATION DE CONFLITS D'INTÉRÊTS}

Les auteurs déclarent n'avoir aucun lien d'intérêt.

\section{Références}

1. Department of economic and social affairs. World economic and social survey 2007: development in an ageing world [En ligne]. New York: United Nations publication; 2007. [cité le 19 juin 2019]. Disponible: https://www.un.org/en/development/desa/policy/ wess/wess_archive/2007wess.pdf
2. Höpflinger F, Bayer-Oglesby L, Zumbrunn A. La dépendance des personnes âgées et les soins de longue durée. Scénarios actualisés pour la Suisse [En ligne]. Berne: Verlag Hans Huber; 2011. [cité le 19 juin 2019]. Disponible: https://www.obsan.admin.ch/sites/ default/files/publications/2015/2011_hh_pflegebed_f.pdf

3. Pefoyo AJ, Bronskill SE, Gruneir A, Calzavara A, Thavorn K, Petrosyan $\mathrm{Y}$, et al. The increasing burden and complexity of multimorbidity. BMC public health. 2015 Apr;(15):415. doi: 10.1186/s12889-0151733-2.

4. Observatoire suisse de la santé. La santé en Suisse - Le point sur les maladies chroniques [En ligne]. Rapport national sur la santé 2015. Berne: Hogrefe Verlag; 2015. [cité le 19 juin 2019]. Disponible: https://www.obsan.admin.ch/sites/default/files/ publications/2015/rapportsante_2015_f_0.pdf

5. Roth S, Pellegrini S. Virage ambulatoire. Transfert ou expansion de l'offre de soins ? [En ligne]. Neuchâtel: Observatoire suisse de la santé; 2015. [cité le 19 juin 2019]. Disponible: https:// www.obsan.admin.ch/sites/default/files/publications/2015/ obsan_68_rapport_0.pdf

6. Organisation mondiale de la Santé (OMS). Rapport mondial sur le vieillissement et la santé. [En ligne]. Genève: OMS; 2016. [cité le 19 juin 2019]. Disponible: https://apps.who.int/iris/bitstream/ handle/10665/206556/9789240694842_fre.pdf?sequence=1

7. Krieg C, Hudon C, Chouinard MC, Dufour I. Individual predictors of frequent emergency department use: a scoping review. BMC Health Serv Res. 2016 Oct;16(1):594. doi: 10.1186/s12913-016-1852-1.

8. Adamuz J, Gonzalez-Samartino M, Jimenez-Martinez E, Tapia-Perez M, Lopez- Jimenez MM, Ruiz-Martinez MJ, et al. Care Complexity Individual Factors Associated With Hospital Readmission: a Retrospective Cohort Study. J Nurs Scholarsh. 2018 Jul;50(4):41121. doi: 10.1111/jnu.12393.

9. Yoshida S, Matsushima M, Wakabayashi H, Mutai R, Murayama $\mathrm{S}$, Hayashi T, et al. Validity and reliability of the Patient Centred Assessment Method for patient complexity and relationship with hospital length of stay: a prospective cohort study. BMJ Open. 2017 May;7(5):e016175. doi: 10.1136/bmjopen-2017-016175.

10. Peek CJ, Baird MA, Coleman E. Primary care for patient complexity, not only disease. Fam Syst Health. 2009 Dec;27(4):287-302. doi: 10.1037/a0018048.

11. Morin E. Introduction à la pensée complexe. Paris: Seuil; 2005.

12. Nightingale F. Notes on nursing: what it is, and what it is not. NewYork: D. Appleton and Compagny; 1989.

13. Fawcett J. The metaparadigm of nursing: present status and future refinements. J Nurs Sch. 1984 Jun;16(3):84-9.

14. Pepin J, Ducharme F, Kerouac S. La pensée infirmière. 4e éd. Montréal: Chenelière éducation; 2017.

15. Richard L, Gendron S, Cara C. Modélisation de la pratique infirmière comme système complexe : une analyse des conceptions de théoriciennes en sciences infirmières. Aporia. 2012 Oct;4(4):25-39.

16. Engel GL. The clinical application of the biopsychosocial model. Am J Psychiatry. 1980 May;137(5):535-44.

17. Bodenheimer T, Wagner EH, Grumbach K. Improving primary care for patients with chronic illness: the chronic care model, part 2. JAMA. 2002 Oct;288(15):1909-14. 
18. Haute Autorité de Santé. Note méthodologique et de synthèse documentaire. Coordination des parcours. Comment organiser l'appui aux professionnels de soins primaires ? [En ligne]. SaintDenis La Plaine, France: HAS; 2014. [cité le 17 septembre 2019]. Disponible: https://www.has-sante.fr/portail/upload/docs/ application/pdf/2014-09/note_appui_coordo_25_09_14.pdf

19. Mount JK, Massanari RM, Teachman J. Patient care complexity as perceived by primary care physicians. Fam Syst Health. 2015 Jun;33(2):137-45.

20. Shippee ND, Shah ND, May CR, Mair FS, Montori VM. Cumulative complexity: a functional, patient-centered model of patient complexity can improve research and practice. J Clin Epidemiol. 2012 Oct;65(10):1041-51.

21. Valderas JM, Starfield B, Sibbald B, Salisbury C, Roland M. Defining comorbidity: implications for understanding health and health services. Ann Fam Med. 2009 Jul-Aug;7(4):357-63.

22. Loeb DF, Binswanger IA, Candrian C, Bayliss EA. Primary care physician insights into a typology of the complex patient in primary care. Ann Fam Med. 2015 Sept;13(5):451-5.

23. Manning E, Gagnon M. The complex patient: a concept clarification. Nurs Health Sci. 2017 Mar;19(1):13-21.

24. Wilson T, Holt T, Greenhalgh T. Complexity science: complexity and clinical care. BMJ. 2001 Sep;323(7314):685-8.

25. De Jonge P, Huyse FJ, Stiefel FC. Case and care complexity in the medically ill. Med Clin North Am. 2006 Jul;90(4):679-92.

26. Stiefel FC, de Jonge P, Huyse FJ, Guex P, Slaets JP, Lyons JS, et al. "INTERMED": a method to assess health service needs. II. Results on its validity and clinical use. Gen Hosp Psychiatry. 1999 JanFeb;21(1):49-56.

27. Pratt R, Hibberd C, Cameron IM, Maxwell M. The Patient Centered Assessment Method (PCAM): integrating the social dimensions of health into primary care. J Comorb. 2015 Jul;5:110-9.

28. Joyce PR, Khan A, Jones AV. The revolving door patient. Compr Psychiatry. 1981 Jul-Aug;22(4):397-403.

29. Busnel C, Marjollet L, Perrier-Gros-Claude O. Complexité des prises en soins à domicile : développement d'un outil d'évaluation infirmier et résultat d'une étude d'acceptabilité. Revue francophone internationale de recherche infirmière. 2018 Jun;4(2):116-23.
30. Morris JN, Fries BE, Steel K, Ikegami N, Bernabei R, Carpenter $\mathrm{Gl}$, et al. Comprehensive clinical assessment in community setting: applicability of the MDS-HC. J Am Geriatr Soc. 1997 Aug;45(8):1017-24.

31. Busnel C, Mastromauro L, Zecca W, Ludwig C. Le RAI Home Care : utilisation, potentiels et limites dans les soins à domicile. Gerontol Soc. 2017 Feb;39(153):167-82.

32. Clark LA, Watson D. Constructing validity: basic issues in objective scale development. Psychol Assess. 1995 Sept;7(3):309-19.

33. Huyse FJ, Lyons JS, Stiefel FC, Slaets JP, de Jonge P, Fink P, et al. "INTERMED": a method to assess health service needs. I. Development and reliability. Gen Hosp Psychiatry. 1999 JanFeb;21(1):39-48

34. Hallgren KA. Computing Inter-Rater Reliability for Observational Data: an Overview and Tutorial. Tutor Quant Methods Psychol. 2012 Jul;8(1):23-34.

35. Cicchetti DV. Guidelines, criteria, and rules of thumb for evaluating normed and standardized assessment instruments in psychology. Psychol Assess. 1994 Dec;6(4):284-90.

36. Landis JR, Koch GG. The measurement of observer agreement for categorical data. Biometrics. 1977 Mar;33(1):159-74.

37. Lobo E, Rabanaque MJ, Bellido ML, Lobo A. Reliability of INTERMED Spanish version and applicability in liver transplant patients: a cross-sectional study. BMC Health Serv Res. 2011 Jul;11:160.

38. Peters LL, Boter H, Slaets JP, Buskens E. Development and measurement properties of the self assessment version of the INTERMED for the elderly to assess case complexity. J Psychosom Res. 2013 Jun; 74(6):518-22.

39. Ludwig C, Busnel C. Protocol of a case-control longitudinal study (fraXity) assessing frailty and complexity among Swiss home service recipients using interRAI-HC assessments. BMC Geriatrics. 2019 Aug;19(1):207. doi:10.1186/s12877. 019-1230-z. 